\title{
The Development of Parameter Estimation Method for Chinese Hamster Ovary Model using Black Widow Optimization Algorithm
}

\author{
Nurul Aimi Munirah ${ }^{1}$, Muhammad Akmal Remli ${ }^{2}$, Noorlin Mohd $\mathrm{Ali}^{3}$, Hui Wen Nies ${ }^{4}$ \\ Mohd Saberi Mohamad ${ }^{5}$, Khairul Nizar Syazwan Wan Salihin Wong ${ }^{6}$ \\ Faculty of Computing, Universiti Malaysia Pahang, 26600, Pekan, Pahang, Malaysia ${ }^{1,3}$ \\ Institute for Artificial Intelligence and Big Data, Universiti Malaysia Kelantan \\ City Campus, Pengkalan Chepa, Kota Bharu 16100, Kelantan, Malaysia ${ }^{2,5,6}$ \\ Malaysia Japan International Institute of Technology (MJIIT) \\ Universiti Teknologi Malaysia, Kuala Lumpur 54100, Malaysia ${ }^{4}$
}

\begin{abstract}
Chinese Hamster Ovary (CHO) cells are very famous in biological and medical research, especially in the protein production industry. It is because the characteristic of the cells with low chromosome numbers make it suitable for genetic study. However, all the data tends to be noisy and not fit. That is why many parameter estimation methods have been developed since their first introduction to determine the best value for a particular parameter. Metaheuristic parameter estimation is an algorithm framework that is processed using some technique to generate a pattern or graph. It will help the researcher get the fitted graph model, correct data, and estimate the value based on the data's behaviour. This process started with implementing the parameter estimation that can be generated by using the combination of mathematical models and all the data obtained from the researcher's experiments. This way, biomedical research's cell culture can benefit from all this metaheuristic parameter estimation used. A kinetic model can estimate the data obtained from the Chinese Hamster Ovary (CHO) cells. Therefore, this paper proposed a Black Widow Optimisation (BWO) algorithm inspired by the bizarre mating behaviour of a spider as the method to use to solve the problem. The proposed algorithm has been compared with the other three famous algorithms, which are Particle Swarm Optimization (PSO), Differential Evolutionary (DE), and Bees Optimization Algorithm (BOA). The results showed that the proposed algorithm could get better value in terms of the best cost despite taking a long time to use.
\end{abstract}

Keywords-Chinese Hamster Ovary; Black Widow optimization; metaheuristic; parameter estimation; genetic study

\section{INTRODUCTION}

\section{A. Background}

Biotechnology, in the most specific word, is a combination of biology with technology. It is a process of modifying biological systems that are found in an organism to make them adaptable in others field. Within this process, bioinformatics will need many research and development process to take place in a laboratory. Industrial biotechnology is also known as white biotechnology because it is a technology that used renewable sources and living cells microorganisms such as bacteria, yeasts, and cells. The researcher uses various techniques to create and improve the collected enzymes [1]. They use the DNA obtained from the experiments to find the enzyme's catalytic capabilities. This technology also makes less waste during manufacturing and production. It is also widely used in industrial sectors like chemicals, pharmaceuticals, and food industries. Fig. 1 shows the development of industrial biotechnology [2]. The most commonly used biotechnology is in the protein production industry under genetic engineering. The process of protein production will need a researcher to manipulate the genes from an organism such as a hamster and make them compatible to use, mainly producing a pharmaceutical product [3].

The kinetic model is one of the tools used in biotechnology. It can represent the biochemistry of cells that is more complex and utterly different from other models. Those models are necessary used to understand and analyse before they can be used industrially. A metaheuristic is an algorithm framework that has been upgraded to a high-level procedure. It is designed to find and generate useful heuristics that may solve the independent problem or an optimisation problem [4]. It will also provide the researcher with smart guidelines or strategies to develop a suitable algorithm. Since Grover introduced the terms in 1986, there are so many types of metaheuristics have been set [4].

Parameter estimation is also knowns as coefficients and sample statistics. It is a technique or process using sample data collected from various fields, such as engineering and biology. Parameter estimation is a descriptive measure for a population. It is also in charge of the response changes related to changing predictors even for one - unit change while other predictors stay constant. It is estimated by using the least square estimations. However, the values are unknown to measure the whole population as it is infeasible. Therefore, the main goal of statistical analysis is to obtain the number of errors parallel with the parameters used. Parameter estimation is usually used to estimate parameters for a selected distribution that is processed using sample data or a number of populations from any potential interest. Also, parameter estimation can determine the best value of parameters by 
going through data assimilation or other similar techniques [5]. Parameter estimation also refers to selecting the best deals from data assimilation for a particular parameter in a numerical model format. With parameter estimation in kinetic models, it is necessary to implement an optimisation method for reducing the distance between estimation models and sample data. Two different ways can be used, which are local and global optimisation. Each method rates based on their minimal best value for each experiment. The local optimisation method has many drawbacks as it can easily be stuck in local minima, that is why the metaheuristic algorithm has the most efficient global optimisation introduced. Hence, most objective functions have several local minima [6].

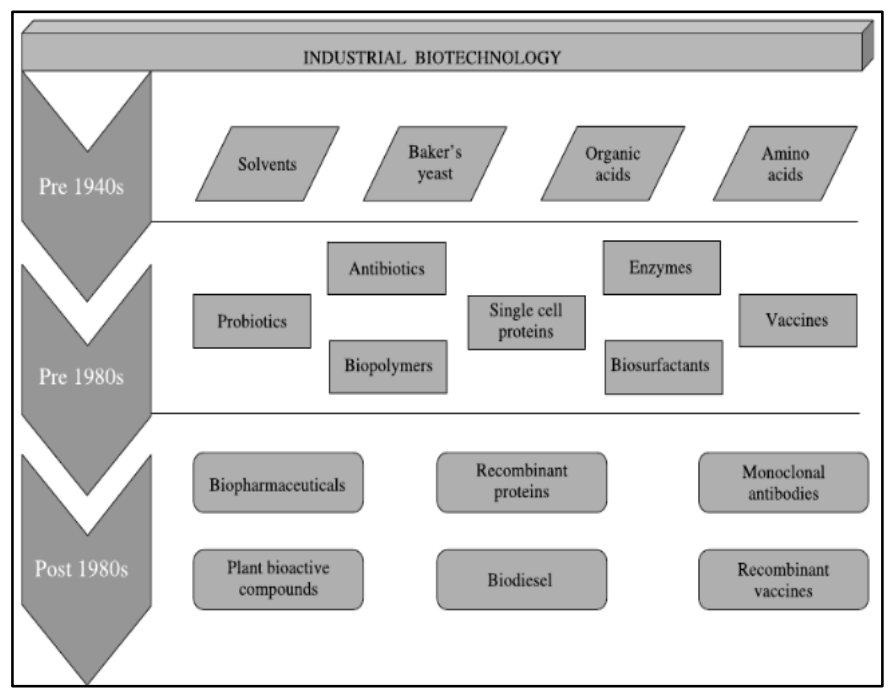

Fig. 1. Development of Industrial Biotechnology.

The metaheuristic parameter estimation for Chinese Hamster Ovary ( $\mathrm{CHO}$ ) research uses a type of metaheuristic to estimate the results of the parameter involved in the $\mathrm{CHO}$ cells. This research will develop a kinetic model that is suitable for the sample data to get the required graphs [7]. Nowadays, the researcher uses the benefits of technologies and becomes more appreciated towards software developed by the software developer. They learn how to use the software, and the results are outstanding. Besides, they use MATLAB to generate the graphs to show the right prediction towards the research.

This research focuses on developing the parameter estimation method to test $\mathrm{CHO}$ sample data using the Black Widow Optimisation (BWO) algorithm. The measurement used is Root - Mean - Square - Error to train the existing sample data. This research also emphasises on comparing the performance of the proposed algorithm and existing metaheuristic optimisation algorithms.

\section{B. Problem Formulation}

Genetic engineering is a complex industry because it will involve a complex number of interacting reactions and regulations [8]. That is one of many main reasons researchers nowadays become more focused on using mathematical models to solve the problems. The estimation used can reduce the scale of error and noise in terms of the distance between the prediction of the model and the data used in the experiments. Many equations represent parameter estimation used to estimate the parameters for each selected sample data such as Probability Plotting, Maximum Likelihood Estimation, and Bayesian Estimation Method. However, this paper will focus on Mean - Square - Error (MSE) as this is denoted how close must regression line be to a set of points. This squaring method is essential to deduct any negative signs. This calculation shows that when the squared error values are getting smaller, the closer the best fit of the line is found. Below is the equation [9]:

$M S E=\left[\frac{1}{n} \sum_{i=1}\left(y_{i}-\bar{y}_{i}\right)^{2}\right.$

Where $\Sigma$ denoted as the summation of all numbers from $(\mathrm{i}=1)$ to $\mathrm{n}$, next, take the $\mathrm{y}$ - coordinate and subtract with $\mathrm{y}_{-}^{-} \mathrm{i}$ - coordinate value before calculating the square of the results. Last, take the sum of $\left(y-y_{-}^{-} i\right) 2$ and start to divide it by $n$. That is how to get the mean, and it is crucial to minimise the value of the mean so that the best lines can be achieved [9].

Therefore, the new forms of algorithm framework and techniques that can be used to the sample of collected data have been using by modern researchers nowadays. The researcher will get accurate prediction results based on their existing data, especially when implementing the right parameter estimation suitable for their research scopes. It can give them an early preview of how the research will work in the future. The graph that will be generated will ease the researcher to make a prediction even in seconds. Therefore, the nearer the line of the graph with the data dotted by the researcher means the minimisation of time has happened, so the better the research's results will be. Not only that, but the metaheuristic used will also help all the biomedical researchers with the kinetic model generated by using those parameter estimation approaches [10-12]. There were many kinds of optimisation algorithms in this world. Still, all of these algorithms have different competency levels as there are algorithms that are not stable in convergence, stuck at local optima, and difficult to tune the parameters.

Thus, a study on Black Widow Optimization (BWO) Algorithm has been developed to test the CHO sample data, and it is inspired by the mating behaviour of black widow spiders. This algorithm has been proved its ability to maintain the balance between the exploitation process and exploration process, which can provide fast convergence and prevent local optima problems.

\section{MetAheuristic OptimisAtion AlgORITHM}

A metaheuristic is a high-level procedure designed to search, generate, and select a technique developed to solve the problems more quickly and search for a solution that classical methods failed to do. Metaheuristic algorithms can be divided into two types, which are single solution and populationbased. Single solution metaheuristic focuses on modifying and improving a solution with a single candidate, while the population-based will maintain and improve multiple solutions for candidates. It will enhance a particular search method to get a better solution. 
Metaheuristic algorithms is also an efficient global optimisation method that, for its search process, it can be divided into two other types which are exploitation and explorations. Exploitation is a search process that depends on information that is already obtained from the problem. It is used to develop a better solution and suitable to be used in the local search method. Although it has a very high convergence rate, it is elementary to stuck at local minima [1]. While for explorations, it will explore the search space more broadly, that is why it can find global solutions even though it is far from the initial point. Hence, the process will be much slower in terms of convergence rate and high in computational cost.

The existing metaheuristic algorithms are summarised their advantages and disadvantages in Table I. Hence, these algorithms will be compared according to their advantages and disadvantages throughout the experiment, in order to achieve more best values.

\section{A. Particle Swarm Optimization (PSO)}

Particle swarm optimisation (PSO) is one of the wellknown metaheuristics ever developed. It is a method to iteratively optimise the problem by improving the solution with a quality measure that has been given. PSO also represents the movement of organisms such as in a bird flock or fish school. Thus, it is meant to simulate those organisms' social behaviour in a way to search for food [13].

A bunch of particles will be used to represent PSO, and it is called a "swarm." Each of the particles is moving based on the influence of its best-known positions. All of those represented particles are freely move around and allowed to search space [14]:

1) Each and own particle will hold its own previous velocity. (Inertia).

2) The distance from its individual particles position. (Cognitive Force).

3) The distance from swarms' position. (Social Force).

Therefore, PSO is a method that can make an assumption or not making any as the problem will be optimised, and the searching can involve a larger space of its candidate solutions. However, PSO will not guarantee in finding the optimal solution.

\section{B. Differential Evaluation (DE)}

Differential Evaluation (DE) is an optimisation algorithm that has been introduced in 1996 by Storn and Price. It is a technique used to optimise a problem by improving its candidate solutions depending on the quality measurements [15]. DE will maintain a population and create new solutions by combining them with its formulae. The best core of solutions will be kept. Therefore, the optimisation problem is a black box that is providing when needed. The use of the DE makes the search adaptable during an evolutionary process [15].

At the initial stage, the parents are far from each other, making the perturbations larger. However, when the process becomes matured, all of the population will converge throughout small regions and make perturbations short [16].
TABLE I. ADVANTAgES AND DisAdVANTAGES OF EXISTING METAHEURISTIC ALGORITHMS

\begin{tabular}{|c|c|c|}
\hline Algorithms & Advantages & Disadvantages \\
\hline $\begin{array}{l}\text { Particle swarm } \\
\text { optimisation } \\
\text { (PSO) }\end{array}$ & $\begin{array}{l}\text { - } \quad \text { Can be simple to } \\
\text { implement } \\
\text { - } \quad \text { Have a few parameters } \\
\text { to adjust } \\
\text { - } \quad \text { Able to run the parallel } \\
\text { computation. } \\
\text { - Can be robust. }\end{array}$ & $\begin{array}{l}\text { - Can be challenging } \\
\text { to define initial } \\
\text { design parameters. } \\
\text { All solutions } \\
\text { converge } \\
\text { prematurely }\end{array}$ \\
\hline $\begin{array}{l}\text { Differential } \\
\text { Evaluations } \\
\text { (DE) }\end{array}$ & $\begin{array}{ll}\text { - } & \text { Better Explorations } \\
\text { - } & \text { Better diversification. } \\
\text { - } & \text { Easy to use }\end{array}$ & $\begin{array}{l}\text { - } \\
\text { cot stable in } \\
\text { convergence. } \\
\text { Need tuning in } \\
\text { parameters }\end{array}$ \\
\hline $\begin{array}{l}\text { Bees } \\
\text { Optimisation } \\
\text { Algorithm } \\
\text { (BOA) }\end{array}$ & $\begin{array}{l}\text { - } \quad \text { Easy to implement } \\
\text { - } \\
\text { sble to perform both } \\
\text { searches (local and } \\
\text { global) }\end{array}$ & $\begin{array}{ll}\text { - } & \text { Need a manual } \\
\text { setting of } \\
\text { parameters. } \\
\text { Has only a few } \\
\text { tuning parameters. }\end{array}$ \\
\hline
\end{tabular}

$\mathrm{DE}$ is worked when it has the candidate solutions that are also called agents in a population. Those agents will move freely around the search space before combining itself with existing agents' positions if the new agents bring an improvement to be kept and accepted into the populations. In contrast, for not accepted agents, they will be discarded. All those processes will be repeated but not guaranteed.

\section{Bees Optimisation Algorithm (BOA)}

Bees Optimisation Algorithm (BOA) is the social behaviour of honeybees to search for food. This algorithm was developed in 2005 [17]. It measures the distance between the defined solutions. BOA will go through local and global search, but BOA will not use any probability approach. It will use a fitness evaluation to proceed with the search process.

BOA, in other words, also has the local and global search that will utilise the exploitation and exploration process [18]. The fitness value will then be checked and sorted descending or going through a process called maximisation problems. The local method will cover the fittest location as its best locations and classify it into two subgroups known as elite and nonelites' sites. The local process will start by recruiting the bees in the elite and non-elite places. The global search will then randomly run the search process before the location is sorted to their fitness value, and global optimum appeared [17].

\section{BLACK WIDOW OPTIMISATION (BWO)}

In this paper, a new set of algorithms has been proposed called Black Widow Optimization (BWO) algorithm, which implements from nature. This algorithm gets the idea from the observations of the black widow spider's unique mating behaviour. Australian Black Widow, also known as redback spider (Latrodectus Hasselti), originated from South Australia or adjacent Western Australian deserts [19]. Now, this spider has also been migrated throughout Australia, Southeast Asia, and New Zealand. A significant difference between a female spider and a male spider is that the adult female spider would be bigger than the male spider. That is why the female spider can reach 10 millimetres, and the male spider is only $3-4$ millimetres long [20]. The adult female spider has a red stripe on the abdomen's upper side at her spherical shape body. 
The mating behaviour's uniqueness can be seen when the female black widow would consume the male black widow, and this behaviour would be called Sexual Cannibalism [21]. Sexual Cannibalism will happen when a female eats a male either before, during, or immediately after mating. For the black widow's case, the adult female spider would mark a particular spot at her net with pheromone hormone. This pheromone would act as a smell that can attract male spiders to come to their trap. After that, the female spider will bite the male spider with her two fangs and inject a complex venom to its prey that definitely would kill them. The female spider will then wrap the male spider with silk before she sucked out all the liquid inside the male spider. This process will help the female spider lay 4 to 10 eggs sacs containing around 250 eggs [22]. The process does not stop here, as the process of Cannibalism will proceed among spiderlings. It is known as Sibling Cannibalism [19]. The spiderlings will consume each other while they live together in their maternal web before being carried away by the wind. This behaviour obviously will affect the population level; however, it is good to raise parental fitness as the survivors have an enhanced body condition.

Lastly, for the unfertilised spiderlings, it is also recorded that they will eat their mother slowly, but this does not happen frequently. This behaviour is called as Matriphagy behaviour. Therefore, several factors caused all of these cannibalistic behaviours:

- Being competitive among each other.

- Lack of food source.

BWO algorithm is used to estimate the parameter of Chinese Hamster Ovary (CHO) cells. This optimisation will use a black widow's behaviour to develop the algorithms to find the best solutions that minimise the nonlinear least-square value that determines the differences between model prediction and experimental data. From the basic concept of the black widow's mating behaviour, a pseudo-code has been developed to give a simple explanation [20], shown in Table II. The process will generate the initial population of the spider and evaluate each spider's fitness. Next, we will decide either we want to end the condition or proceed with the situation. If we move with the problem, it will start with randomly selecting the parents before procreation happens as they will try to reproduce the new generation of spiders in pairs. After that, the process of Cannibalism will take place before the mutation process. Lastly, the population of the spiders will be updated.

\section{A. Initial Population}

The optimisation problem can be solved with appropriate values of problem variable structure, and for the BWO algorithm, it is known as "widow." Each of the black widow spiders will show the problem variables, and for this paper, the structure will be represented as an array [20].

For the dimensional optimisation problem [Nvar] the solution for the problems will be represented by the widow as an array [1 $x$ Nvar]. This array would be defined as follows[1]:
Widow $=\left[x_{1}, x_{2}, \ldots\right.$, Nvar $]$

All of the variable values $\left[x_{1}, x_{2}, \ldots, N v a r\right]$ will act as a floating-point number. Therefore, the evaluation of fitness function $f$ will be helpful in order to the fitness value of the widow.

Fitness $=f($ widow $)=f\left[x_{1}, x_{2}, \ldots\right.$, Nvar $]$

In order to start the optimisation algorithm, the initial populations of spiders are very important in terms of generating the candidate widow matrix [Npop $x$ Nvar] before performing the procreating step via mating by randomly selected pairs of parents.

\section{TABLE II. PSEUDO - CODE OF BWO ALGORITHM}

Input Maximum number of iterations, Rate of procreating,

Rate of Cannibalism, Rate of mutation

Output Near-optimal solution for the objective function

\section{// Initialization}

1 The initial population of black widow spiders

Each pop is a D-dimensional array of chromosomes for a D-dimensional problem

\section{// Loop until the terminal condition}

2 Based on the procreating rate, calculate the number of reproductions "nr"

3 Select the best nr solutions in pop and save them in pop1

\section{// Procreating and Cannibalism}

4 For $\mathrm{i}=1$ to $n r$ do

5 Randomly select two solutions as parents from pop1

6 Generate D children using Equation 1

7 Destroy father

8 Based on the cannibalism rate, destroy some of the children (new achieved solutions)

9 Save the remain solutions into pop2

10 End For

\section{// Mutation}

11 Based on the mutation rate, calculate the number of mutation children "nm"

12 For $\mathrm{i}=1$ to $\mathrm{nm}$ do

13 Select a solution from pop1

14 Randomly mutate one chromosome of the solution and generate a new solution

15 Save the new one into pop3

16 End For

// Updating

17 Update pop $=$ pop2 + pop3

18 Return the best solution

19 Return the best solution from pop 


\section{B. Procreate}

The spider will start to mate here as to generate new generations of the spiders. Researches show that each mating process will produce around 1000 eggs, but only the strongest and fit baby spiders will be survived[1]. Now with the presence of random numbers of widow array, an array named alpha will be created so the offspring that have been produced will be using $\alpha$.

$x_{1}$ and $x_{2}=$ parents $\& y_{1}$ and $y_{2}=$ offspring

$y_{1}=\alpha \times x_{1}+(1-\alpha) \times x_{2}$

$y_{1}=\alpha \times x_{2}+(1-\alpha) \times x_{1}$

This process should not be duplicated but must be repeated for $\left[\frac{N v a r}{2}\right]$ times. Then, the spiders, both mother and children will be added to the array and be sorted according to their fitness value.

\section{Cannibalism}

There were three kinds of Cannibalism involved in this algorithm[1]:

\section{1) Sexual Cannibalism}

- The female black widow will eat her husband during or after mating process.

- The fitness value can determine their gender.

2) Sibling Cannibalism

- The competitions between spiderlings occurred as the stronger spiderlings would eat the weaker spiderlings.

- The survivors among siblings would be set as Cannibalism Rating (CR).

3) Matriphagy

- It rarely happened but still being observed.

- The unfertilised spiderling will eat their mother slowly.

- The quality of the spiderlings, whether strong or weak, can be determined using fitness value.

\section{Mutation}

The mutation process will begin by selecting Mutepop as the number of individuals randomly. Two elements in the array would be randomly exchanged by each chosen solution. The mutation rate that has been calculated called Mutepop. Fig. 2 shows the flow of BWO Mutation.

\section{E. Convergence}

There are three stop conditions that can be considered, and I choose (i) as I define the iterations and can see later that BWO will have better results compared to other algorithms:

1) The predefined number of iterations.

2) Observe the unchanged fitness value for several iterations.

3) Reached the level of accuracy that has been specified.

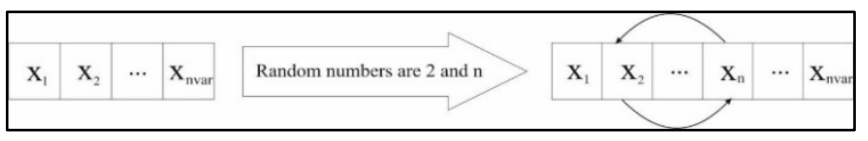

Fig. 2. BWO Mutation.

\section{F. Parameter Setting}

Some parameters need to be taken care of to get better results. These parameters need to be adjusted well to improve the algorithm's success rate. The right parameter values are very important to control the exploitation balance and stages of explorations.

1) Procreating Rate (PP)

- The percentage of procreating.

- Determine the number of individuals involved to procreate.

- Control the offspring productions and provide more chances to explore the search space.

2) Cannibalism Rate (CR)

- Acts as a controller to the cannibalism operator that would deduct unfit individuals from populations.

- High performance of the exploitation stage can be ensured by adjusting the parameter with proper values.

3) Mutation Rate (PM)

- The individuals participate during mutations in percentage.

- Control transferring between search agents from the global and local stage.

- Propelling towards best solutions.

\section{EXPERIMENTAL SETUP}

\section{A. Dataset}

In this research, Chinese Hamster Ovary (CHO) cells have been used as the datasets as it is widely used in the biological engineering process. $\mathrm{CHO}$ has already been used in research since 1919. It is because of CHO cells' characteristics compared to other cells regarding their rapid growth in suspension culture and high protein production [5]. Therefore, the $\mathrm{CHO}$ cells are useful for radiation cytogenetics and tissue culture. Table III shows the details of the CHO model used in this research.

CHO cells are also used in protein production for fermentation processes [16]. These cells will not be fed for 12.5 days, equal to 300 hours. This process is called the simulation batch process using resting cells. As mentioned above, the cells are usually used in the fermentation process, which has already become industrial to make products useful for the human being. Therefore, the fermentation process involves some metabolic processes that will act on its enzymes by making chemical changes to its organic substrate. This industrial fermentation has been applied to the food industry and the general industry to produce commonly used chemical concentrations such as citric acid, ethanol, and 
lactate. All of these cells' concentration will be affected by the rate of fermentation. The fermentation process will need a fermenter medium to supply all of those nutrients needed by the organisms or cells. Examples of sources involved (including carbon and amino acid) are glucose and leucine or methionine, respectively. Therefore, the fermenter process will measure 13 metabolites, such as glucose, lactate, product protein, leucine, methionine, cytosol, aspartate, malate, pyruvate, oxaloacetate, ATP, and ADP. All of these samples will be taken daily during the whole fermentation process.

In CHO, 35 metabolites are comprised and divided into three compartments: fermenter, cytosol, and mitochondria. Thirty-two reactions are generated in $\mathrm{CHO}$ that included protein product formation (the process of connecting the protein using peptide bonds and the process of forming the polypeptide from amino acids substance), Embden Meyerhof Parnas Pathway (the conversion of glucose using the metabolic pathway), TCA cycle, amino acid metabolism reduction, production of lactate, and electron transport chains. Therefore, the ordinary differential equation (ODE) model produced will comprise 117 parameters and ensure that the purpose of doing optimisation by generating pseudo experimental data and mimicking typical cells' behaviour is achieved.

TABLE III. CHO MODEL USED IN THIS RESEARCH

\begin{tabular}{|l|l|l|l|l|}
\hline Model ID & Cell & Description Level & Parameter & Data Type \\
\hline $\begin{array}{l}\text { Chinese } \\
\text { Hamster } \\
\text { Ovary }\end{array}$ & CHO & Metabolic & 117 & Simulated \\
\hline
\end{tabular}

\section{B. Comparisons of Methods Experiment}

The best values of all the algorithms used, including BWO, PSO, DE, and BOA, were evaluated. The CPU times were also compared to show which algorithm takes longer times. All of them were tested with CHO sample data. Thus, this work was performed using Matlab R2015b.

\section{RESUlts AND DisCUSSION}

In this section, the Black Widow Optimization (BWO) algorithm has been tested with a constant number of iterations set to 100 iterations. All of these experiments are timeconsuming and need excellent computational systems for it to work well. The results obtained from the experiments will be compared with other existing metaheuristics, which are Particle Swarm Optimization (PSO), Differential Evaluation (DE), and Bee Optimization Algorithm (BOA). Table IV presents all of the parameters involved in experiments for 20 runs.

An initial population of the spider has been generated and then evaluates each spider's fitness. Continue with the decision making, either the condition is ended or proceeded. The crossover process or procreating then happens, so the second population of spiders is generated. After that, the process of mutation generates the third population will take place. These crossover and mutation are significant because they can start to reproduce the new generation of the spiders by uniting all the populations that have been generated. Then, beginning to sort the spider population is based on its fitness value to determine the best cost of the BWO algorithm with 117 dimensions (nVar). Lastly, all the extra individuals are deleted before the population of the spiders is updated. Hence, the results are always different from each time of the experiments run because of the problem's stochastic nature. The results are reported in Table V. The table shows the experiments' results between BWO, PSO, DE, and BOA for 117 dimensions. According to the table, BWO, which always has the lowest best value, can be considered the best algorithm compared to the others despite its not having the shortest CPU Times. As a result, BWO can be claimed can converge better. Therefore, there were several advantages and disadvantages of BWO [20], shown in Table VI.

\section{A. Comparison In Graph (Best Cost by 100 Iteration)}

Fig. 3, 4, 5 and 6 below shows the graph of each experimental algorithm from BWO, PSO, DE and BOA for its best cost by 100 iterations.

\section{B. Comparison In Graph (Best Cost by CPU Times)}

Fig. 7, 8, 9, and 10 below shows the graph of each experimental algorithm from BWO, PSO, DE and BOA for its best cost by CPU Times.

TABLE IV. PARAMETERS AND VALUES INVOLVED IN EXPERIMENTS

\begin{tabular}{|l|l|l|}
\hline Algorithms & Parameters & Values \\
\hline \multirow{4}{*}{ BWO } & Percent of Crossover = pc (Procreating Rate) & 0.60 \\
\cline { 2 - 3 } & Cannibalism Rate = pCannibalism & 0.44 \\
\cline { 2 - 3 } & $\begin{array}{l}\text { Percent of Mutation = pMutation (Mutation } \\
\text { Rate) }\end{array}$ & 0.40 \\
\hline \multirow{4}{*}{ PSO } & Inertia Weight = w & 2.00 \\
\cline { 2 - 3 } & Best personal Experience = c1 & 2.40 \\
\cline { 2 - 3 } & Best Global = c2 & 2.20 \\
\cline { 2 - 3 } & Intertia Weight Damping Ratio = wdamp & 0.98 \\
\hline \multirow{3}{*}{ DE } & Mutation Factor = beta & 0.50 \\
\cline { 2 - 3 } & Crossover Constant = pCR & 0.20 \\
\hline \multirow{2}{*}{ BOA } & Neighborhood Radius Damp Rate = rdamp & 0.95 \\
\hline
\end{tabular}

TABLE V. RESULTS

\begin{tabular}{|l|l|l|l|}
\hline Algorithms & Iteration & Best Cost & CPU Times \\
\hline$B W O$ & 100 & 22488.5989 & 6288.8125 \\
\hline$P S O$ & 100 & 155817.0963 & 1714.3438 \\
\hline$D E$ & 100 & 2992715.1241 & 2577.6563 \\
\hline$B O A$ & 100 & 4703432.5252 & 20781.25 \\
\hline
\end{tabular}

TABLE VI. ADVANTAgES AND DisAdVANTAgES OF BWO

\begin{tabular}{|l|l|}
\hline Advantages & Disadvantages \\
\hline $\begin{array}{l}\text { Provide better results in exploitation and } \\
\text { exploration stages. }\end{array}$ & New optimisation algorithm. \\
\hline Deliver fast convergence speed. & Does not fully exposed yet. \\
\hline $\begin{array}{l}\text { Able to check the large area to get the best } \\
\text { solutions. }\end{array}$ & \\
\hline
\end{tabular}




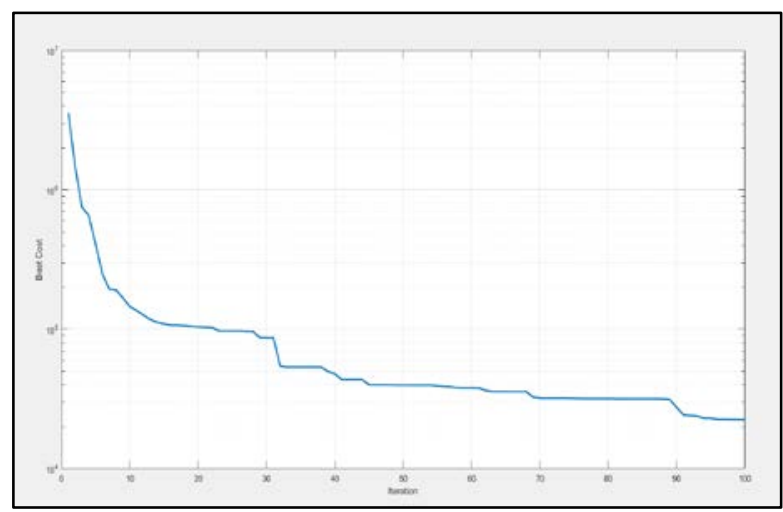

Fig. 3. The Best Cost of BWO by 100 Iteration.

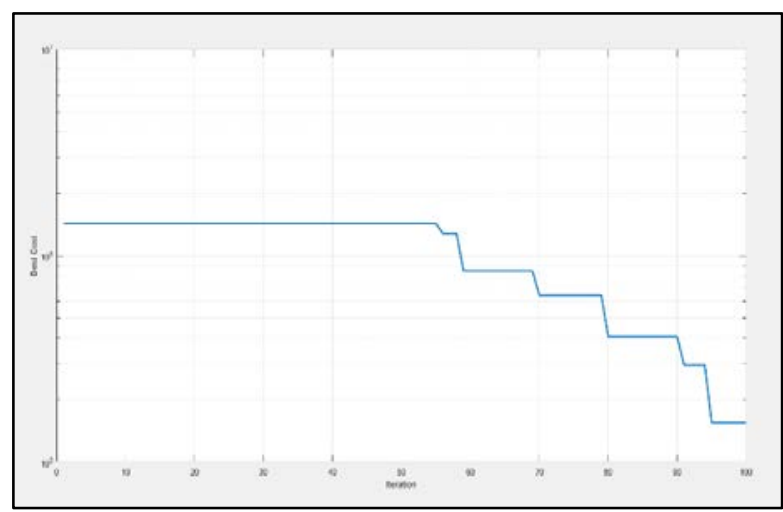

Fig. 4. The Best Cost of PSO by 100 Iterations.

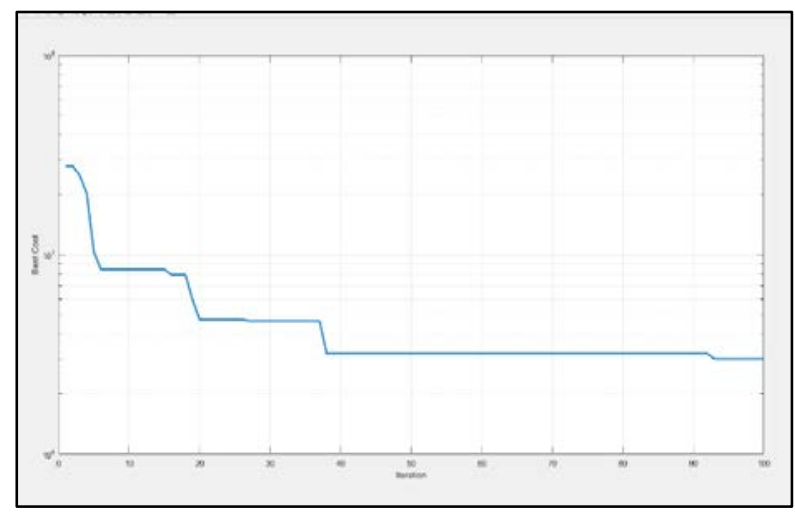

Fig. 5. The Best Cost of DE by 100 Iterations.

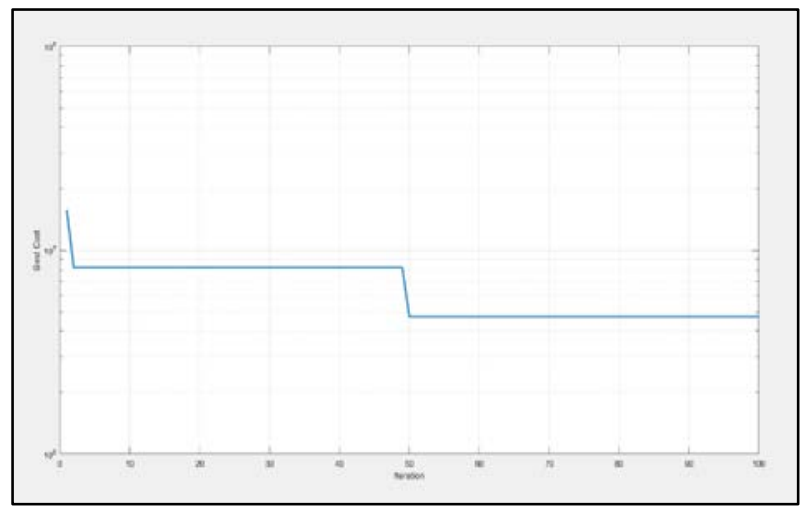

Fig. 6. The Best Cost of BOA by 100 Iterations.

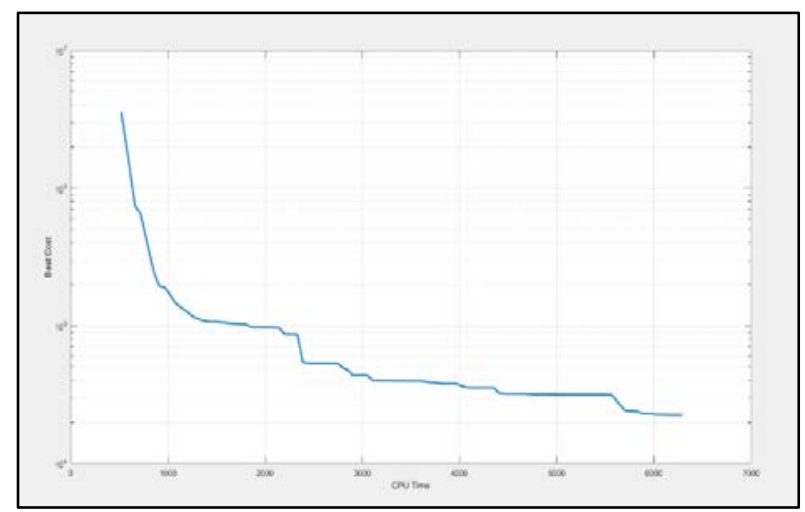

Fig. 7. The Best Cost of BWO by CPU Times.

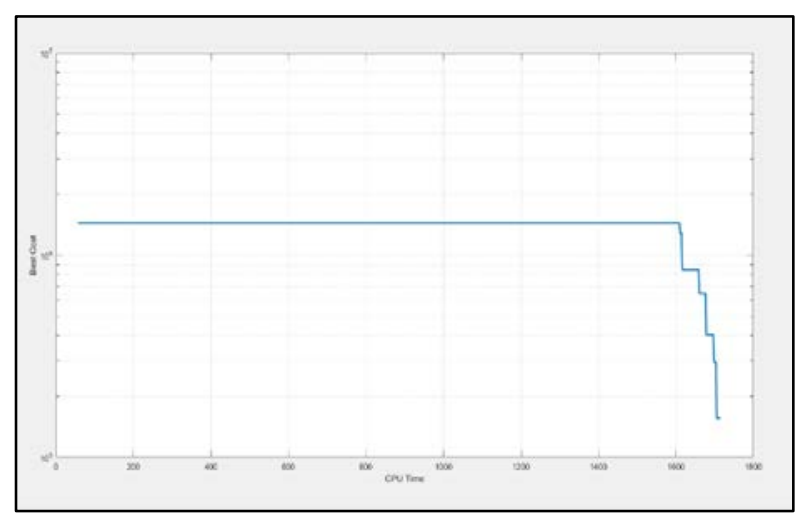

Fig. 8. The Best Cost of PSO by CPU Times.

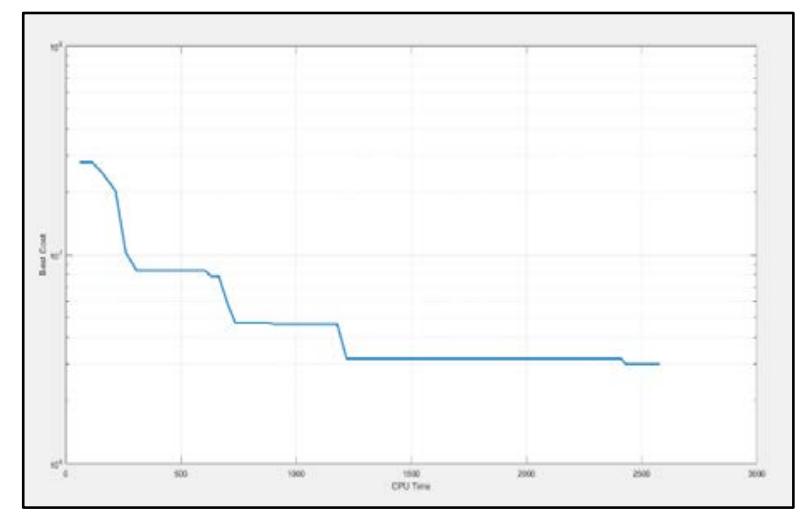

Fig. 9. The Best Cost of DE by CPU Times.

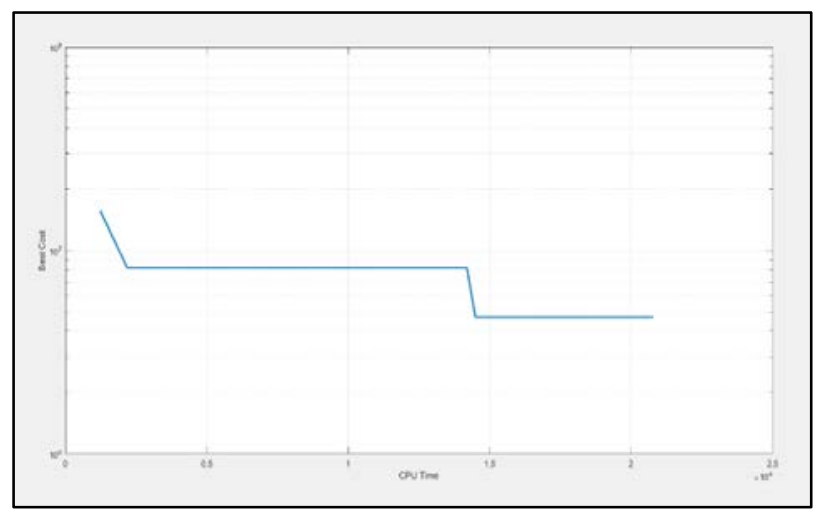

Fig. 10. The Best Cost of BOA by CPU Times. 


\section{CONCLUSIONS}

In conclusion, this paper discussed the new algorithms called Black Widow Optimization (BWO), inspired by a venomous spider's unique and extreme mating behaviour. This algorithm has been studied with three other algorithms, which are Particle Swarm Optimization (PSO), Differential Evolutionary (DE), and Bees Optimization Algorithm (BOA). It has shown high performance to find the most nearer optimal values that are accurate and fast in converging. According to the results, BWO can be a useful contribution to solving the larger optimisation scale problem. The proposed algorithm would be limited in terms of the metabolic field and can contribute to real-world optimisation problems and be applied to various areas. Besides, randomly selecting the parents of the spider in BWO has ensured the exploration process. At the same time, offspring produced in the procreate step already emphasises it and help prevent traps in local optima. The solutions that have been omitted during the cannibalism stage also help BWO algorithms work faster to achieve the best solutions. Lastly, the BWO algorithm can be considered to be a smart algorithm to solve optimisation problems.

This research has a few limitations. Although BWO shows obtained the best value in terms of the best cost, this algorithm sometimes takes to finish the experiments compared to other algorithms in this study: PSO, DE, and BOA. Second, this research also needs a good quality of PC or laptop processor to ensure that the software users do not lag and stop working suddenly.

For future study, BWO could be tested with more other metaheuristics and different kinds of datasets. Also, trying the BWO with a larger iteration is to ensure fitness.

\section{ACKNOWLEDGEMENT}

We would like to thank Malaysia Ministry of Higher Education for their support via Fundamental Research Grant Scheme [FRGS/1/2018/ICT02/UMP/02/8] and Fundamental Research Grant Scheme - Malaysia Research Star Award [R/FRGS/A0800/01655A/003/2020/00720].

\section{REFERENCES}

[1] J. Almquist, M. Cvijovic, V. Hatzimanikatis, J. Nielsen, and M. Jirstrand, "Kinetic models in industrial biotechnology - Improving cell factory performance,” Metabolic Engineering, vol. 24. Academic Press Inc., pp. 38-60, 01-Jul-2014.

[2] "10 Everyday uses of Biotechnology | CPI.” [Online]. Available: https://www.uk-cpi.com/blog/10-everyday-uses-of-biotechnology. [Accessed: 20-Jul-2020].

[3] “(PDF) Industrial Biotechnology: An Overview.” [Online]. Available: https://www.researchgate.net/publication/311576484_Industrial_Biotech nology_An_Overview. [Accessed: 19-Jul-2020].

[4] G. G. Wang, S. Deb, and Z. Cui, "Monarch butterfly optimisation," Neural Comput. Appl., vol. 31, no. 7, pp. 1995-2014, 2019.

[5] M. A. Remli, S. Deris, M. S. Mohamad, S. Omatu, and J. M. Corchado, "An enhanced scatter search with combined opposition-based learning for parameter estimation in large-scale kinetic models of biochemical systems,” Eng. Appl. Artif. Intell., vol. 62, pp. 164-180, Jun. 2017.

[6] "Parameter Estimation.” [Online]. Available: https://sphweb.bumc. bu.edu/otlt/MPH-

Modules/BS/BS704_Confidence_Intervals/BS704_Confidence_Intervals 2.html. [Accessed: 20-Jul-2020].

[7] J. Y. Kim, Y. G. Kim, and G. M. Lee, "CHO cells in biotechnology for production of recombinant proteins: Current state and further potential," Applied Microbiology and Biotechnology, vol. 93, no. 3. pp. 917-930, Feb-2012.

[8] "genetic engineering | Definition, Process, \& Uses | Britannica." [Online]. Available: https://www.britannica.com/science/geneticengineering. [Accessed: 20-Jul-2020].

[9] "Machine learning: an introduction to mean squared error and regression lines.” [Online]. Available: https://www.freecodecamp.org/news/ machine-learning-mean-squared-error-regression-line-c7dde9a26b93/. [Accessed: 20-Jul-2020].

[10] A. F. Villaverde et al., "BioPreDyn-bench: A suite of benchmark problems for dynamic modelling in systems biology,” BMC Syst. Biol., vol. 9, no. 1, pp. 1-15, 2015.

[11] Remli MA, Mohamad MS, Deris S, Sinnott R, Napis S. An Improved Scatter Search Algorithm for Parameter Estimation in Large-Scale Kinetic Models of Biochemical Systems. Current Proteomics. 2019 Oct 1;16(5):427-38.

[12] Kunna MA, Kadir TA, Remli MA, Ali NB, Moorthy K, Muhammad N. An Enhanced Segment Particle Swarm Optimization Algorithm for Kinetic Parameters Estimation of the Main Metabolic Model of Escherichia Coli. Processes. 2020 Aug;8(8):963.

[13] X. S. Yang and M. Karamanoglu, "Swarm Intelligence and Bio-Inspired Computation: An Overview," in Swarm Intelligence and Bio-Inspired Computation, Elsevier Inc., 2013, pp. 3-23.

[14] Z. Cui and X. Gao, "Theory and applications of swarm intelligence," Neural Computing and Applications, vol. 21, no. 2. pp. 205-206, Mar2012.

[15] R. Storn and K. Price, "Differential Evolution - A Simple and Efficient Heuristic for Global Optimisation over Continuous Spaces," J. Glob. Optim., vol. 11, no. 4, pp. 341-359, 1997.

[16] S. Mirjalili, S. M. Mirjalili, and A. Lewis, “Grey Wolf Optimizer,” Adv. Eng. Softw., vol. 69, pp. 46-61, 2014.

[17] D. T. Pham and M. Castellani, "A comparative study of the Bees Algorithm as a tool for function optimisation," Cogent Eng., vol. 2, no. 1, Dec. 2015.

[18] B. Yuce, M. S. Packianather, E. Mastrocinque, D. T. Pham, and A. Lambiase, "Honey bees inspired optimisation method: The bees algorithm," Insects, vol. 4, no. 4, pp. 646-662, Nov. 2013.

[19] F. F. S. Daly, R. E. Hill, G. M. Bogdan, and R. C. Dart, "Neutralisation of Latrodectus mactans and $\mathrm{L}$. hesperus venom by redback spider ( $\mathrm{L}$. hasseltii) antivenom,” J. Toxicol. - Clin. Toxicol., vol. 39, no. 2, pp. 119-123, Jan. 2001.

[20] V. Hayyolalam and A. A. Pourhaji Kazem, "Black Widow Optimization Algorithm: A novel meta-heuristic approach for solving engineering optimisation problems," Eng. Appl. Artif. Intell., vol. 87, p. 103249, Jan. 2020.

[21] J. E. Garb, A. González, and R. G. Gillespie, “The black widow spider genus Latrodectus (Araneae: Theridiidae): Phylogeny, biogeography, and invasion history,” Mol. Phylogenet. Evol., vol. 31, no. 3, pp. 11271142, 2004.

[22] O. R. Pires, W. Fontes, and M. S. Castro, "Recent Insights in Latrodectus ('Black Widow' Spider) Envenomation: Toxins and Their Mechanisms of Action,” in Spider Venoms, Springer Netherlands, 2016, pp. 333-344. 